\title{
Primer registro del Mosquero Coronado Empidonomus aurantioatrocristatus (Tyrannidae) al occidente de Ecuador
}

\author{
William A. Arteaga-Chávez \\ Universidad Central del Ecuador, Facultad de Ciencias Biológicas, Carrera de Biología, Quito, Ecuador. \\ Correo electrónico: arteagawilliam95@yahoo.es \\ Editado por/Edited by: Juan Freile \\ Recibido/Received: 12 agosto 2020. Aceptado/Accepted: 05 marzo 2021 \\ Publicado en línea/Published online: 16 mayo 2021
}

First record of Crowned Slaty Flycatcher Empidonomus aurantiotroacristatus (Tyrannidae) in western Ecuador

\begin{abstract}
Crowned Slaty Flycatcher Empidonomus aurantioatrocristatus is an austral migrant that winters in western Amazonia. In this note, I present the first record west of the Andes in Ecuador, of a bird found at El Morán, province of Carchi. One individual observed in December 2019 was aged as first-year by plumage, and likely corresponds to an accidental vagrant given date and location.
\end{abstract}

Keywords: Austral migrant, Carchi, vagrant, wintering area.

\section{Resumen}

El Mosquero Coronado Empidonomus aurantioatrocristatus es un migrante austral que pasa el invierno al oeste de la Amazonía. Presento el primer registro de esta especie al oeste de los Andes ecuatorianos, en El Morán, provincia de Carchi. Un individuo observado en diciembre de 2019 presentaba un plumaje de primer año. Dada la fecha y lugar de registro, este individuo debe corresponder a una presencia accidental.

Palabras clave: Área de invernada, Carchi, migratorio austral, vagabundo.

El Mosquero Coronado Empidonomus aurantioatrocristatus comprende dos subespecies distribuidas ampliamente al este de los Andes de América del Sur, entre 0-1100 m s.n.m. (Robb, 2020; Remsen et al., 2020). La subespecie E. a. pallidiventris es residente en el centro-este de Brasil (Somenzari et al., 2018), mientras que E. a. aurantioatrocristatus se reproduce en el norte y este de Bolivia, centro-oeste y sur de Brasil, norte y centro de Argentina, Paraguay y Uruguay durante el verano austral (Robb, 2020). Esta subespecie migra al norte de Sudamérica durante la temporada no reproductiva, y pasa el invierno austral principalmente al oeste de la Amazonía, en el noreste de Perú, este de Ecuador, sureste de Colombia, regiones de Manaos y Belém en Brasil, sur de Venezuela y sur de Guyana (Somenzari et al., 2018; Robb, 2020).

En Ecuador, E. a. aurantioatrocristatus reside durante el invierno austral (marzo-septiembre) en las tierras bajas del este (Ridgely \& Greenfield, 2001; Freile \& Restall, 2018). Es poco común y mayormente solitario; ocupa áreas abiertas con árboles dispersos, en ecosistemas de tierra firme y bordes de bosque de igapó y várzea (Freile \& Restall, 2018). La mayoría de registros se encuentra bajo los 1100 m s.n.m., pero existe un reporte en Cuyuja a 1600 m s.n.m y uno Vilcabamba a 2600 m s.n.m. (Fig. 1) (Ridgely \& Greenfield, 2001; Freile \& Restall, 2018). En esta nota, presento el primer registro de E. a. aurantioatrocristatus en la vertiente occidental de los Andes de Ecuador y brevemente discuto otros reportes extra-límite. 


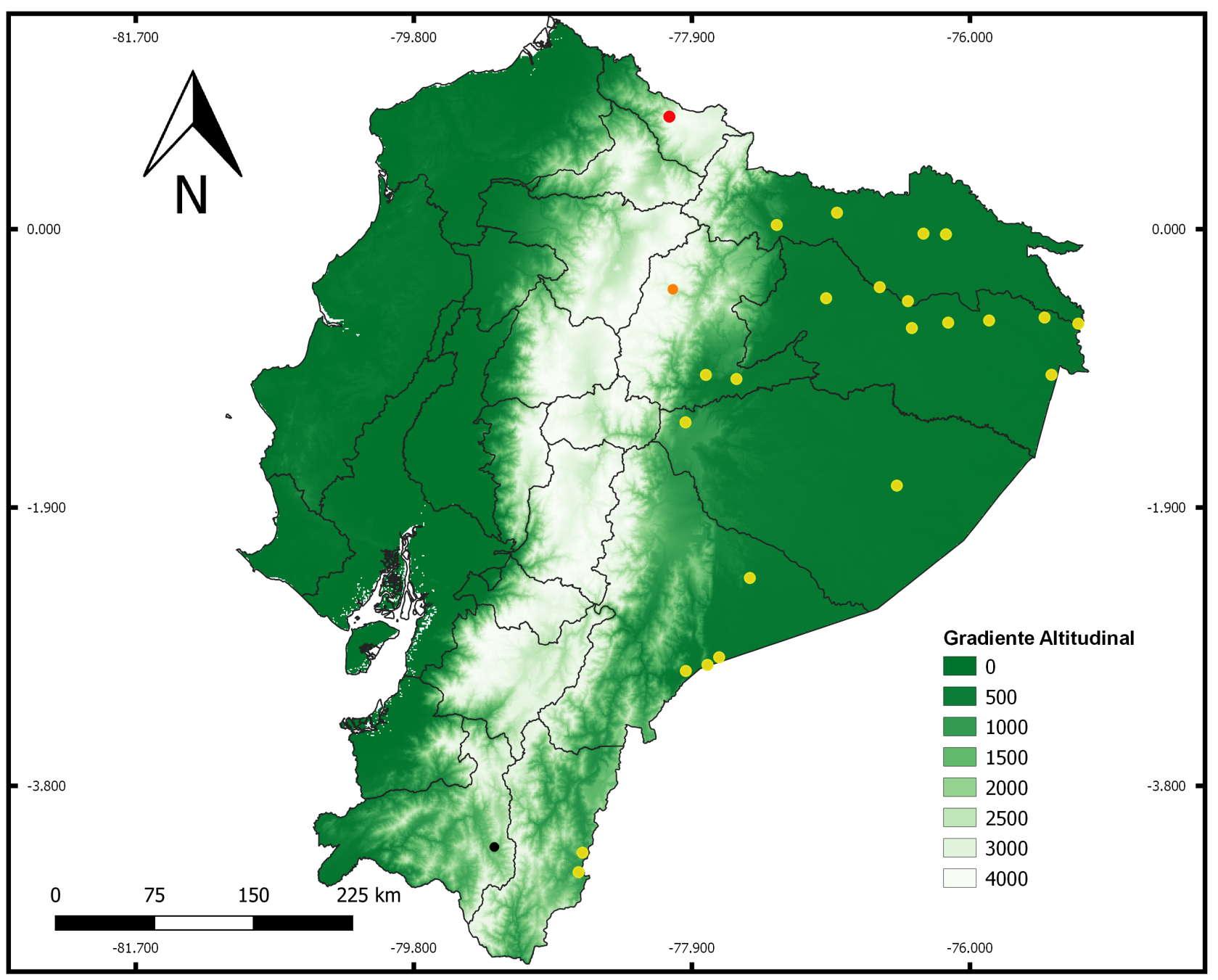

Figura 1. Registros del Mosquero Coronado Empidonomus aurantioatrocristatus en Ecuador. En amarillo, localidades dentro de su rango habitual de distribución (Freile \& Poveda, 2019; eBird, 2020); en naranja (Cuyuja, Napo) y negro (Vilcabamba, Loja), registros previos fuera área de distribución en el este de los Andes (Ridgely \& Greenfield, 2001; Freile \& Poveda, 2019); en rojo, nueva localidad en los Andes occidentales.

El 21 de diciembre de 2019 observé y fotografié un individuo de E. a. aurantioatrocristatus (Fig. 2) en la comunidad El Morán (0,768175, -78,054109; 2816 m s.n.m.), noroccidente de la provincia de Carchi, durante el XII Conteo Navideño Binacional de Aves Chiles-Chical. El individuo voló y se perchó por $c$. 2 min en una cerca de alambre a 1,5 m del suelo en un área abierta con pastizales y parches de matorrales dispersos cerca del poblado.

Schulenberg et al. (2008) y Robb (2020) señalan que los adultos tienen un parche amarillento semioculto en medio de la corona negra y, en general, tienen coloración gris parduzca uniforme, aunque con el dorso más opaco y las partes ventrales más claras. El individuo observado y fotografiado tenía un tamaño aproximado de $17 \mathrm{~cm}$ y las marcas más notorias de campo eran la corona pardo-negruzca, ceja pálida muy tenue y franja orbital negruzca desde los lores. El dorso gris parduzco era deslucido, flancos mayormente grises y vientre levemente amarillento, contrastando con el pecho más oscuro (Fig. 2). En las alas se notaba una barra blanquecina en las coberteras medianas y márgenes pálidos en el vexilo externo de las plumas secundarias. De acuerdo con Schulenberg et al. (2008) y Robb (2020), esta característica del plumaje corresponde a individuos juveniles, por lo que se infiere que el ejemplar registrado era un inmaduro en su primer año. Normalmente, determinar una edad aproximada en esta especie es complejo, debido a que puede presentar un plumaje desgastado como resultado de la migración o puede haber experimentado algún tipo de muda en el área de invernada. Si bien podría confundirse con individuos inmaduros del Mosquero Variegado Empidonomus varius o del Mosquero Pirata Legatus leucophaius, E. a. aurantioatrocristatus se diferencia de estas dos especies porque carece de bigotera pálida o malar negruzco en todas sus fases de plumaje, y su espalda y pecho son siempre más llanos (Schulenberg et al., 2008). 


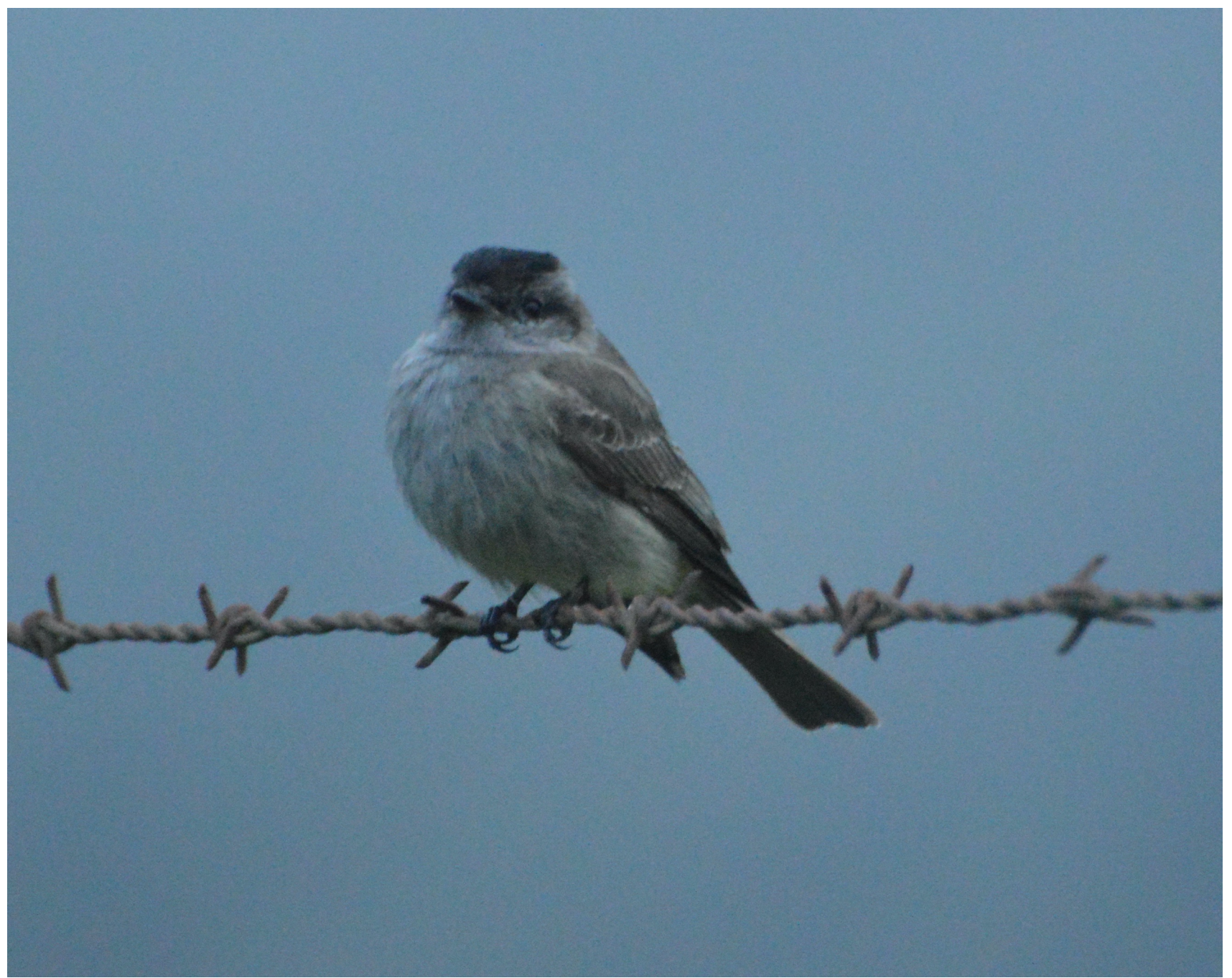

Figura 2. Mosquero Coronado Empidonomus aurantioatrocristatus en El Morán, noroccidente de la provincia del Carchi, Ecuador, 21 de diciembre de 2019 (William A. Arteaga-Chávez).

Existen varios reportes septentrionales fuera del rango habitual de migración de esta especie. Se considera accidental al norte del río Orinoco, en los estados de Amazonas y Mérida, Venezuela, con un espécimen colectado en febrero de 1950 en Paso Portachuelo, centro-norte de Aragua (Hilty, 2003). En la plataforma continental de América del Sur, el registro más al norte proviene de Tayrona, Colombia (Burris, 2019). Tayrona y Paso Portachuelo son las localidades continentales más cercanas al océano Atlántico, aunque existe un reporte en la isla Bonaire, al norte de las costas venezolanas (Schets, 2018).

El reporte a mayor altitud proviene de los Andes bolivianos, a 3300 m s.n.m. (Chesser, 1995). Adicionalmente, existen cuatro registros de individuos que han cruzado la cordillera de los Andes; dos de ellos provienen de la vertiente Pacífica de los Andes: Calama, Chile (Jaramillo, 2003) y Vilcabamba, sur de Ecuador, aunque esta última se encuentra cerca del paso de cordillera hacia la vertiente amazónica (Ridgely \& Greenfield, 2001). Los otros dos son extra-límite: uno en Cerro Azul, Panamá (Robb et al., 2009) y el más septentrional en Louisiana, Estados Unidos (Conover \& Myers, 2009).

Esta observación en Carchi constituye el primer reporte de E.a.aurantioatrocristatus en la vertiente occidental de los Andes de Ecuador, y sugiere que la especie podría cruzar los Andes con más frecuencia de lo esperado. Existen dos probables rutas de llegada al oeste de Carchi. El individuo pudo desviarse de su ruta migratoria al retornar al sur y cruzar los Andes ecuatorianos en algún paso relativamente bajo. Alternativamente, pudo cruzar los Andes en el extremo norte de su área de invernada, en Colombia, y retornar hacia el sur por las tierras bajas o piemontanas del occidente. El Morán se encuentra $116 \mathrm{~km}$ al oeste de la localidad más cercana dentro su área de distribución en Ecuador (Lumbaqui, Sucumbíos) y representa el registro a mayor altitud en el país (Freile \& 
Restall, 2018). Similar a registros previos de otros Tyrannidae migratorios australes, el individuo observado corresponde a un vagabundo que se quedó a pasar el verano austral en el norte, debido a que en diciembre la especie debería estar en sus zonas de reproducción (Robb, 2020).

Empidonomus aurantioatrocristatus y E. varius son especies simpátricas. La última también habita en gran parte de América del Sur, al este de los Andes, y su subespecie nominal migra hacia la Amazonía occidental durante el invierno austral (Mobley \& Kirwan, 2020). Existen escasas observaciones de individuos vagabundos de E. varius fuera de su área de invernada (Howell et al., 2014). Sin embargo, se han documentado cuatro registros en América del Norte: uno al oeste (Mlodinow \& Irons, 2009) y dos al este de Estados Unidos (Abbott \& Finch, 1978; Nicholson \& Steadman, 1988), y uno en el sureste de Canadá (Houle \& Houle, 1993).

Algunos reportes extra-límite de especies migratorias australes o de migrantes inversos corresponden a individuos de primer año (Howell et al., 2014; Schets, 2018; Burris, 2019). Con el reporte aquí presentado, son dos los registros de individuos de E.a.aurantioatrocristatus de primer año y alrededor de 12 fuera de temporada o área de invernación (Jaramillo, 2003; Robb et al., 2009; Schets, 2018). De forma general, estas observaciones sugieren que E. a. aurantioatrocristatus tiene cierta tendencia a vagabundear o permanecer fuera de su zona normal de distribución invernal con más frecuencia que su congénere E. varius.

\section{AGRADECIMIENTOS}

A la Fundación Altrópico por el soporte logístico en el XII Conteno Navideño Binacional de Aves ChilesChical. Karlla Barbosa compartió información especializada. Juan Freile y José María Loaiza amablemente revisaron el manuscrito y proporcionaron valiosos comentarios. Dos revisores anónimos sugieron comentarios importantes para la estructuración del manuscrito.

\section{REFERENCIAS}

Abbott, D. J., \& Finch, D. W. (1978). First Variegated Flycatcher (Empidonomus varius) record for the United States. American Birds, 32, 161-163. URL: https://sora.unm.edu/sites/default/files/journals/nab/v032n02/ p00161-p00163.pdf

Burris, C. T. (2019). Northernmost mainland South American record of Crowned Slaty Flycatcher Empidonomus aurantioatrocristatus at Tayrona National Natural Park, Colombia. Cotinga, 41, 111-112. URL: https://www.neotropicalbirdclub.org/cotinga/C41/Cotinga41-190618-digital-hr.pdf

Chesser, R. T. (1995). Biogeographic, ecological, and evolutionary aspects of South American austral migration, with special reference to the family Tyrannidae (Ph.D. dissertation). Louisiana State University, Baton Rouge.

Conover, P. E., \& Myers, B. M. (2009). First United States record of Crowned Slaty-Flycatcher (Empidonomus aurantioatrocristatus) from Louisiana. North American Birds, 62, 638-639. URL: http://www.losbird.org/lbrc/csfl nab.pdf

eBird. (2020). eBird: an online database of bird distribution and abundance. Ithaca, NY: Cornell Lab of Ornithology. URL: https://ebird.org

Freile, J. F., \& Poveda, C. (2019, Abril 18). Empidonomus aurantioatrocristatus. En: Freile, J. F., Poveda, C. (Eds.), Aves del Ecuador. Versión 2019.0. Quito, Ecuador: Museo de Zoología, Pontificia Universidad Católica del Ecuador. URL: https://bioweb.bio/faunaweb/avesweb/FichaEspecie/Empidonomus\%20aurantioatrocristatus

Freile, J. F., \& Restall, R. (2018). Birds of Ecuador. Londres, U.K.: Helm Field Guides.

Hilty, S. L. (2003) Birds of Venezuela. Princeton, NJ: Princeton University Press.

Howell, S. N., Lewington, I., \& Russell, W. (2014). Rare birds of North America. Princeton, NJ: Princeton University Press. 
Houle, D., \& Houle, J. (1993). First record of Variegated Flycatcher for Canada. Birders Journal, 2, 291-300.

Mlodinow, S. G., \& Irons, D. S. (2009). First record of Variegated Flycatcher for western North America. Western Birds, 40, 47-49. URL: https://sora.unm.edu/sites/default/files/journals/wb/v40n01/wbv40n1-p047-p051.pdf

Mobley, J., \& Kirwan, G. M. (2020). Variegated Flycactcher (Empidonomus varius), version 1.0. In: del Hoyo, J., Elliott, A., Sargatal, J., Christie, D. A. \& de Juana, E. (Eds.), Birds of the World. Ithaca, NY: Cornell Lab of Ornithology. DOI: https://doi.org/10.2173/bow.varfly.01

Nicholson, C. P., \& Steadman, S. J. (1988) The official list of Tennessee birds, Addendum I. Migrant, 59, 1-4.

Remsen, J. V., Areta, J. I., Bonaccorso, E., Claramunt, S., Jaramillo, A., Pacheco, J. F., Ribas, C., Robbins, M. B., Stiles, F. G., Stotz, D. F., \& Zimmer. K. J. (2020, February 05). A classification of the bird species of South America. American Ornithological Society. URL: www.museum.lsu.edu/ Remsen/SACCBaseline.htm

Ridgely, R. S., \& Greenfield, P. J. (2001) The birds of Ecuador. Vol. 1. Status, distribution and taxonomy. Ithaca, NY: Cornell University Press.

Robb, R. (2020). Crowned Slaty Flycatcher (Empidonomus aurantioatrocristatus), version 1.0. In: Schulenberg, T.S. (Eds), Birds of the World. Ithaca. NY: Cornell Lab of Ornithology. DOI: https://doi.org/10.2173/bow.croslf1.01

Robb, R. R., Arendt, D., Larsen, K., \& Sherrell, P. (2009). First North American record of Crowned Slaty Flycatcher Griseotyrannus aurantioatrocristatus, at Cerro Azul, Panama. Cotinga, 31, 50-52. URL: https://www.neotropicalbirdclub.org/wp-content/uploads/2016/10/C31-Robbetal.pdf

Schets, P. P. (2018) Crowned Slaty Flycatcher: another new bird for Bonaire and for the Kingdom of the Netherlands. BioNews, 19, 13. URL: https://www.dcnanature.org/wp-content/uploads/2018/11/BioNews19FlyCatcher.pdf

Schulenberg, T. S., Lane, D. L., O’Neill, J. P., \& Stotz, D. F. (2008). Field identification of juvenile Empidonomus tyrants. Neotropical Birding, 3, 31-36. URL: https://www.neotropicalbirdclub.org/wpcontent/uploads/2020/06/NB3-NB-empidonomus.pdf

Somenzari, M., Amaral, P. P., Cueto, V. R., Guaraldo, A. C., Jahn, A. E., Lima, D. M., Lima, P. C., Lugarini, C., Machado, C. G., Martinez, J., Nascimento, J. L. X., Pacheco, J. F., Paludo, D., Prestes, N. P., Serafini, P. P., Silveira, L. F., Sousa, A. E. B. A., Sousa, N. A., Souza, M. A., Telino-Júnior, W. R., \& Whitney, B. M. (2018). An overview of migratory birds in Brazil. Papéis Avulsos de Zoologia, 58: e20185803. DOI: https://doi.org/10.11606/1807-0205/2018.58.03 